\title{
LINEAR LIST COLORING OF SOME SPARSE GRAPHS
}

\author{
Ming Chen \\ College of Mathematics Physics and Information Engineering \\ Jiaxing University, Zhejiang 314001, China \\ e-mail: chen2001ming@163.com \\ YUSHENG LI \\ AND \\ LI ZHANG ${ }^{1}$ \\ School of Mathematical Sciences \\ Tongji University Shanghai 200092, China \\ e-mail: li_yusheng@tongji.edu.cn \\ lizhang@tongji.edu.cn
}

\begin{abstract}
A linear $k$-coloring of a graph is a proper $k$-coloring of the graph such that any subgraph induced by the vertices of any pair of color classes is a union of vertex-disjoint paths. A graph $G$ is linearly $L$-colorable if there is a linear coloring $c$ of $G$ for a given list assignment $L=\{L(v): v \in V(G)\}$ such that $c(v) \in L(v)$ for all $v \in V(G)$, and $G$ is linearly $k$-choosable if $G$ is linearly $L$-colorable for any list assignment with $|L(v)| \geq k$. The smallest integer $k$ such that $G$ is linearly $k$-choosable is called the linear list chromatic number, denoted by $l c_{l}(G)$. It is clear that $l c_{l}(G) \geq\left\lceil\frac{\Delta(G)}{2}\right\rceil+1$ for any graph $G$ with maximum degree $\Delta(G)$. The maximum average degree of a graph $G$, denoted by $\operatorname{mad}(G)$, is the maximum of the average degrees of all subgraphs of $G$. In this note, we shall prove the following. Let $G$ be a graph, (1) if $\operatorname{mad}(G)<\frac{8}{3}$ and $\Delta(G) \geq 7$, then $l c_{l}(G)=\left\lceil\frac{\Delta(G)}{2}\right\rceil+1 ;(2)$ if $\operatorname{mad}(G)<\frac{18}{7}$ and $\Delta(G) \geq 5$, then $l c_{l}(G)=\left\lceil\frac{\Delta(G)}{2}\right\rceil+1 ;(3)$ if $\operatorname{mad}(G)<\frac{20}{7}$ and $\Delta(G) \geq 5$, then $l c_{l}(G) \leq\left\lceil\frac{\Delta(G)}{2}\right\rceil+2$.
\end{abstract}

Keywords: linear coloring, maximum average degree, planar graphs, discharging.

2010 Mathematics Subject Classification: 05C15.

\footnotetext{
${ }^{1}$ Corresponding author.
} 


\section{REFERENCES}

[1] D.W. Cranston and G. Yu, Linear choosability of sparse graphs, Discrete Math. 311 (2011) 1910-1917.

doi:10.1016/j.disc.2011.05.017

[2] W. Dong, B. Xu and X. Zhang, Improved bounds on linear coloring of plane graphs, Sci. China Math. 53 (2010) 1895-1902. doi:10.1007/s11425-010-3073-0

[3] W. Dong and W. Lin, On linear coloring of planar graphs with small girth, Discrete Appl. Math. 173 (2014) 35-44. doi:10.1016/j.dam.2014.03.019

[4] L. Esperet, M. Montassier and A. Raspaud, Linear choosability of graphs, Discrete Math. 308 (2008) 3938-3950. doi:10.1016/j.disc.2007.07.112

[5] C. Li, W. Wang and A. Raspaud, Upper bounds on the linear chromatic number of a graph, Discrete Math. 311 (2011) 232-238. doi:10.1016/j.disc.2010.10.023

[6] C.H. Liu and G. Yu, Linear colorings of subcubic graphs, European J. Combin. 34 (2013) 1040-1050.

doi:10.1016/j.ejc.2013.02.008

[7] Y. Wang and Q. Wu, Linear coloring of sparse graphs, Discrete Appl. Math. 160 (2012) 664-672.

doi:10.1016/j.dam.2011.10.028

[8] R. Yuster, Linear coloring of graphs, Discrete Math. 185 (1998) 293-297.

doi:10.1016/S0012-365X(97)00209-4

Received 12 May 2017

Revised 31 January 2018

Accepted 31 July 2018 